\title{
Web-based Development Environment and Its Application
}

\author{
Feng Pan \\ School of Information Engineering \\ Guizhou Minzu University \\ Guiyang, China \\ E-mail: panf@vip.163.com
}

\author{
Juan Han \\ School of Foreign Languages \\ Guizhou Minzu University \\ Guiyang, China \\ E-mail: sharonhj@163.com
}

\begin{abstract}
In order to use software development environments conveniently and efficiently, this paper proposes a design scheme for integrated environment for the software development of web-based application patterns and gives instances developed under the environment. This scheme adopts browser/server architecture with Web frameset as its user interface, network intermediate components as its business process center, and uses $\mathrm{I} / \mathrm{O}$ redirection to realize human-machine interaction. This paper implements the sharing of development environments, provides multi-user online use, extends computer environment, thus providing a convenient practical platform for numerous programming learners.
\end{abstract}

Keywords-Integrated development environment;Web application; $\mathrm{I} / \mathrm{O}$ redirection

\section{INTRODUCTION}

With the rapid development and deep application of Internet, the design and application patterns of software are gradually changed. The software development tool, which takes the integrated development environment (IDE) as its mainstream platform, integrates practical and powerful functions such as editing, compiling and debugging, but also presents some disadvantages. Traditional IDE is effective for desktop systems, but not for browsers, which makes it difficult to be shared and used online. IDE is powerful as well as complicated. So for program design that only needs compiling capability, most capabilities of IDE are idle. How to trim complicated IDE , and refine the most basic function , provide an easy-to-use interface so that users can share these functions are the targets of this paper .

From the viewpoint of software users, simplicity, convenience, complete functions and low costs are the basic conditions for choosing software systems. Web-based software systems are public-oriented with excellent crossplatform application features and take Web browser as the standard interface between users and systems. At present, Web-based software engineering has become a research focus of many scholars as well as a hot spot of software engineering design. Literatures [1-8] implemented Webbased software systems and provide a Web-based application environment. This paper, by effectively integrating network intermediate components, provides a simple Web-based software development environment and elaborates on its applications as well as the implementation of key technologies.

\section{SYSTEM DESIGN}

\section{A. The Basic Design Ideas of the System}

System client-side uses Web interface. Users submit programs via a Web form. Server-side is composed of Web server, intermediate components and compiling tools. Hereinto, intermediate components mainly have four functional modules: module for reading and save programs, module for calling compiler to compile programs and capture information about compiling, module for loading running programs and capturing running results, module for analyzing compiling or running results files and giving assessment.

\section{B. System Working Principle}

As shown in Figure 1, users' HTTP requests are submitted to Web server, which calls intermediate components to process the submitted data. The intermediate components first write relevant data to files, then call tools such as compilers or interpreters to process the written file. The information produced by processing uses redirection technology to be written to files. Intermediate component set includes some Ch scripts running in the Ch environment and is written in $\mathrm{C}$ language. The file set here mainly refers to text files, which can be divided into two types: one is the source codes and data files of high-level languages and, which are submitted by Web forms, and then read and generated by intermediate components; the other is results file which are generated by compilers or other command line tools and are STDOUT or STDERR written via redirection technology, then returned to the client-side via Web server.

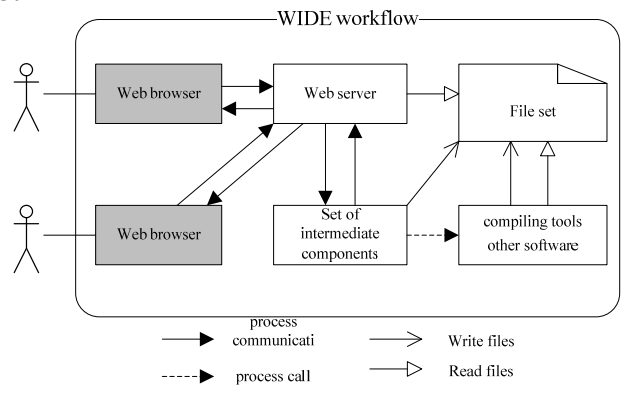

Figure 1. Workflow of the system 


\section{Functional Modules}

\section{1) User Interface Design}

User interface is the interface through which problem solvers communicate with a system. The program for solving problem is submitted via Web forms. The evaluation results that the system gives to programs are also displayed in the client-side browser in the form of Web files. In this paper, we adopt the form of Web frameset. That is, the browser client area is subdivided into several rectangular areas, so that inputs and outputs are conducted in the same interface, which is similar to the integrated development environment. User interface is simply divided into the following three sections:

Selection interface. The interface mainly serves as users' reading area and is used by users to select relevant functions and services. Navigation function is realized mainly by hyper-linking titles.

Interface for editing and title submission. The interface is used as the input area of program submission by users as well as the output area of selection interface, which is realized using embedded Web framework.

Output interface. Like the submission interface, this interface uses embedded Web framework and is displayed mainly as the server-side returned results.

As shown in Figure 2, the selection interface serves as the navigation interface. The pages users select to hyperlink are displayed in the submission interface. And the request results submitted by the submission interface are displayed in the result pages.

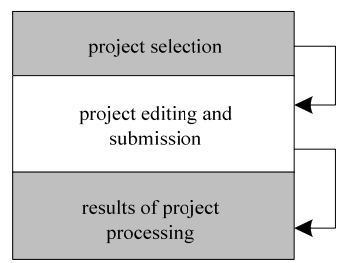

Figure 2. Web framework-based user interface

\section{2) Middleware Design}

The network intermediate component is the business center of the system and can be called by Web server, extending the functions of Web server. It reads HTTP request data and writes the source codes of the program that has been read to files and at the same time calls server-side compiler to compile programs and is loaded into Shell through the compiled programs. The compiling results or running results are redirected to files and return the URL of the results files to client-side so that users can view.

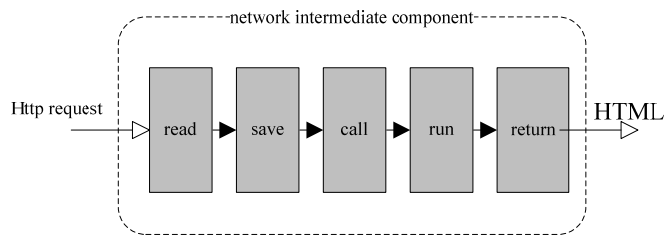

Figure 3. Capabilities of network intermediate component

\section{Key Application Technologies}

The interaction between users and remote software tools is mainly realized through redirection technology. The Shell environment of the operating system supports input and output redirection, which can be expressed by the symbols "<" and ">", respectively. 0, 1 and 2 stand for STDIN, STDOUT and information output of STEERR respectively and can be used to specify the STDIN or STDOUT that needs to be redirected. For example, 2>error.txt means that error information should be output to file error.txt; Using redirection ">", the dir command which originally means viewing the contents, can directly write screen output to a file and view then results in the file. The same is true of input. It can be redirected to a file and the redirection operators can be used to redirect the command input and output dataflow from a default location to other position. The location of input or output dataflow is called handle. There are typically three types of handle: STDIN, STDOUT and STDERR, as shown in Table 1.

TABLE I. STANDARD I/O

\begin{tabular}{|c|c|c|}
\hline Handle & Code of handle & Description of handle \\
\hline STDIN & 0 & Standard input \\
\hline STDOUT & 1 & Standard output \\
\hline STDERR & 2 & Standard error \\
\hline
\end{tabular}

\section{SYSTEM IMPLEMENTATION}

\section{A. Implementation of User Interface}

User interface mainly includes area of navigating page functions, area of editing and submitting, and area of displaying processed results. In the Web page in which the three areas are located, the in-page framework tag $<$ frame name $=$ "AreaName" $></$ frame $>$ is used to indicate the area name. The navigation area uses hyperlink tag $<a$ href= "javaPro.html" target $=" E d i t F r ">\ldots</ a>$ to hyperlink the edit page javaPro.html and display position EditFrame of Java program.

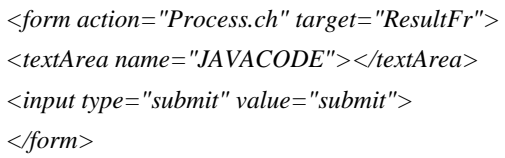

In the above HTML code snippet, <textArea > indicates and edits the text area of codes. The action attribute value Process.ch of the tag $<$ form $>$ is the server-side program of processing and submitting codes. The target attribute value indicates that the results are displayed in the framework in which the attribute value of name is ResultFr.

\section{B. Implementation of Middleware}

Network intermediate component Process.ch is a script written in $\mathrm{C}$ language and it runs in the environment of $\mathrm{Ch}$ virtual machine. The key codes are as follows: 


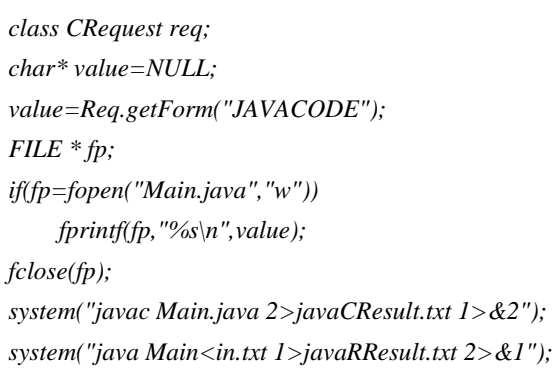

The last two lines in the codes above mean compiling Java program and redirecting the compiled output to file javaCResult.txt, and interpreting and running Java program. And those in file in.txt are the input data required for running. The output results are redirected to file javaRResult.txt.

\section{APPLICATION INSTANCES}

\section{A. Java Program Testing}

As shown in figure 5, we integrate edit area of source programs, the data input when programs run and the display area of running results in a Web page. This transparent design is similar to the integrated development environment of the desktop system.

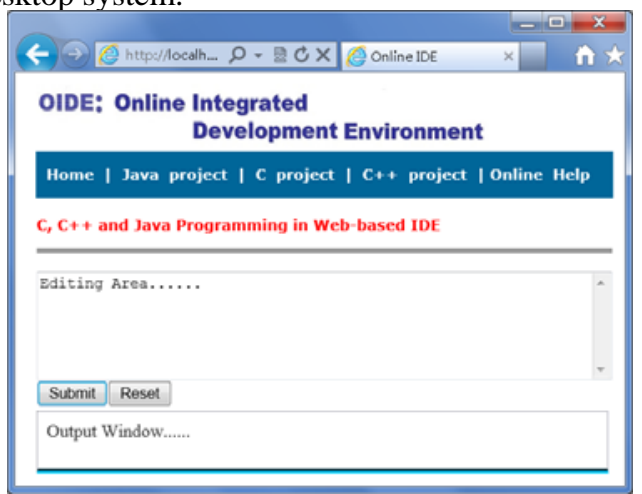

Figure 4. OIDE interface

After selecting Java programs, edit the program and input data before submission. The bottom area of the webpage displays three hyperlinks: hyper-linking source code file; compiling results file and running results file.

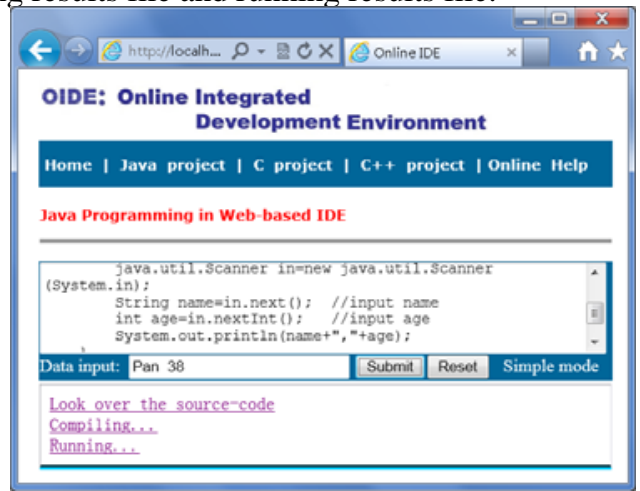

Figure 5. Submission of Java program
If the program and the data input are both correct, choose the hyperlink "Running...", then the running results will be displayed in the output area, as shown in Figure 6; if the program is wrong, for example, the semicolon in the output statement is removed, error of compiling can be viewed via hyperlink "Compiling...", as shown in Figure 7. If the compiling is correct, nothing can be seen in the hyperlink.

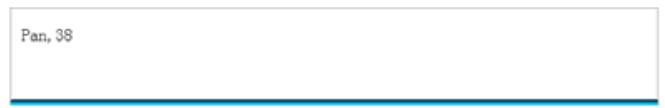

Figure 6. Running results

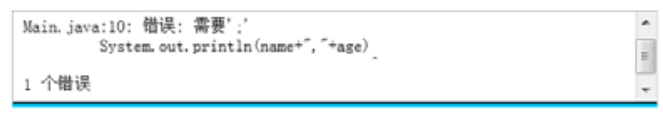

Figure 7. Compiling results

\section{B. OJ System Testing}

OJ System (Online Judge System) is the system for users to submit and evaluate their programs, so as to test the results of problem solving. ACM International Collegiate Programming Contest (ACM/ICPC) also adopts OJ System for strict verification. It is the typical application of WIDE. The prototype of OJ System can be divided into three design topics: design of problems, design of user interface and design of business logics.

1) Design of Problem Library

Title design. The title must explicitly describe the problems to be solved. It is described using natural language, so there must be no ambiguity and the execution capacity of the current system should be fully taken into consideration.

Description of input format. When problem description is correct, the input format of data can help problem solvers to design the correct codes of data input of the programs. The codes must easily read the data used for testing by redirection.

Description of output format. Like those mentioned above, when problem description is correct, the output format of data can help problem solvers to design the correct codes of data output of the programs. The programs must be easily written to the files that save results by redirection.

Test data. Test data are saved in text files and redirected to the programs when programs are running. As the standard input part of programs, each datum is separated using space, Tab character or carriage return in the file.

Standard result data. The programs read test data and take them as standard input when the programs are running. The running output results are written to a text file by redirection. Then the system compares the content of this text file with standard result data, thus judging whether the programs pass the test.

2) Design of Business logics

The user interface of OJ system is similar to the abovementioned instance. Its key part is business logics design, which consists of the following four processing units.
a) reading and saving 
In Web system, client-side submits programs to the server by means of forms typically using POST method and GET method. Due to big data, POST method is used and data are read by standard input equipment. And the data read by standard input equipment are written to text files and saved on the server side as source files.

\section{b) compiling}

Design the compiled command line, take the statements of the command line as the parameter of system function and call system function. The prompts produced by compiling is redirected to file $\mathrm{A}$. If file $\mathrm{A}$ is empty, it compiles and goes to run; or it fails to compile, and then the information about compiling failure will be returned.

\section{c) running}

In the case of successful compiling, the running command line will be built. Just like compiling, the statements of the command line is taken as the parameter of system (char*) function, which will be called. In the running command line, test data is redirected to the program and the running results are redirected to file B.

d) judging

Compare standard result data with file $\mathrm{B}$, if they are consistent, then True is output, otherwise False is output.

\section{Experimental Environment}

The experiment server-side system is Windows Server 2003 and uses IIS (Internet Information Server) to provide Web server capabilities while setting up Ch professional edition as the running environment of Ch scripts. Network intermediate components are Ch scripts that are written in $\mathrm{C}$ language and they are executed in the $\mathrm{C} / \mathrm{C}++$ interpreting and computing environment $\mathrm{Ch}$. The seamless integration of Ch-CGI kit and Web server realizes the interaction between client-side and the server. The setup of JDK1.6 provides tools such as compiler and interpreter of Java program. The compiling of $\mathrm{C} / \mathrm{C}++$ program uses $\mathrm{GUN}$.

\section{COMPARISONS AND DISCUSSIONS}

Programs developed in the Web-based compiling environment run on the remote server-side. And the input and output of data need to be centralized. This design pattern is not suitable for programs that interact frequently. But this pattern enables sharing of the computing resources on the platform and extends workspace, so that we do not need to setup and maintain the system. The compiling results through local computers are usually displayed in the console, and the Web-based compiling environment needs to redirect the results of processing to files, and then returned to clientside via Web server.
The Web-based system needs to consider scale, namely the bearing capacity when a large number of users access the system. But the environment is targeted at a special population and special needs, so the scale is usually not large and the system can bear. To satisfy the large-scale needs only requires the addition of the URL of multiple systems to the Web page of the same portal site. Random links or relevant strategies are adopted to realize the equal access to each system.

\section{CONCLUSIONS}

Web system extends the environment of a computer, which brings convenience to people. Web-based integrated development environment is a favorable extension of traditional integrated development environment. It changes software's design patterns as well as its usage pattern. Software changes from a commodity to a service. Online, sharing, convenience and low costs etc. are the advantages of Web-based system. Focusing on server-side and providing better and practical services are what will be done next.

\section{ACKNOWLEDGMENT}

This study is supported by Natural Science and Technology Foundation of Guizhou Province of China(Guikehe J LKM [2011] No. 18).

\section{REFERENCES}

[1] K. Q. He, R. Peng, W. Liu, J. Wang and B. Li, "Networked Software,” Beijing: Science Press , 2008.

[2] H. H. Cheng, " $\mathrm{C}$ for Engineers and Scientists: An Interpretive Approach,” New York : McGraw-Hill, 2009.

[3] S. Murugesan, "Web Application Development: Challenges and the Role of Web Engineering,” John Karat and Jean Vanderdonckt, eds., Web Engineering, Modelling and Implementing Web Applications, Springer, Berlin, 2008, pp. 7-32.

[4] F. Pan, H. Xi, R. L. Zhang, "A Distributed Platform Design and Implementation of Compiler," Journal of Southwest China Normal University,vol. 35, Aug. 2007, pp.141-144.

[5] W. X. Chen, J. B. Xiang, J. G. Chen, "Web-based Remote Matlab Command Window Design and Implementation," Journal of Computer Applications and Software, vol. 26, Feb. 2009, pp.138-141,

[6] H. H. Cheng, "Ch:A Pedagogically Effective Cross-Platform Interpretive $\mathrm{C} / \mathrm{C}++$ Computing Environment," Journal of Computer Education, Jul. 2009, pp.34-46.

[7] Q. C. Yu, X. J. Weng, Y. Zhao, “An Interactive Open Architecture Computer Vision Platform:Ch OpenCV," Journal of Computer Engineering and Applications, vol.23, Dec. 2006, pp.78-81.

[8] F. Pan, "Mathematical Function Recognition and Its Visualization System Based on Web,” Proc. of the 2011 Int'l Conf. on Computer Science and Service System. IEEE Computer Society, Jun. 2011,pp.1783-1786. 